\title{
An Optimization Approach to Joint Cell and Power Allocation in Multicell Networks
}

\author{
Mikael Fallgren*, Hildur Æsa Oddsdóttir* and Gábor Fodor ${ }^{+}$ \\ Technical Report TRITA-MAT-2011-OS1 \\ Department of Mathematics \\ Royal Institute of Technology \\ January 2011
}

\begin{abstract}
Since the seminal paper by Knopp and Humblet that showed that the system throughput of a singlecell system is maximized if only one terminal transmits at a time, there has been a large interest in opportunistic communications and its relation to various fairness measures. On the other hand, in multicell systems there is a need to allocate transmission power such that some overall utility function is maximized typically under fairness constraints. Furthermore, in multicell systems the degree of resource allocation freedom includes the serving cell selection that allows for load balancing and thereby the efficient use of radio resources. In this paper we formulate the joint serving cell selection (link selection) and power allocation problem as an optimization task whose purpose is to maximize either the minimum user throughput or the multicell sum throughput. The max-min problem and a simplified max throughput problem are both NP-hard and we therefore propose heuristic solution approaches. We present numerical results that give new and valuable insights into the trade off between fair and sum throughput optimal joint resource allocation strategies.
\end{abstract}

\section{INTRODUCTION}

The seminal paper by Knopp and Humblet showed that the system throughput of a singlecell (code division multiple access) system is maximized if only one terminal transmits at a time [1]. This result did not only trigger further research on opportunistic communications, but also found applications in practical systems such as those using the high speed packet access (HSPA) technology [2]. More recently, opportunistic power control (OPC) for multicell systems has been shown to be throughput optimal for data traffic [3]. OPC exploits channel fluctuations such that it increases the transmission power when the channel is good and the transmission rate is adjusted according to the received signal-to-interference-and-noise (SINR) ratio.

Although the OPC concept is attractive because it maximizes the multicell throughput and lends itself for

This research was supported by the Swedish Foundation for Strategic Research (SSF) via the Center for Industrial and Applied Mathematics (CIAM) at KTH and by Ericsson.

*Optimization and Systems Theory, Department of Mathematics, Royal Institute of Technology, Stockholm (werty@kth.se; haodd@kth.se)

+Ericsson Research, Sweden (Gabor.Fodor@ericsson.com) distributed implementations, it requires instantaneous and quite precise channel state information and can become extremely unfair. Indeed, previous works proposed computationally efficient algorithms that deal with the fairness issue of OPC [3], [4], typically at the expense of some loss in the overall throughput. For the uncoded multi-user multiple input multiple output (MIMO) downlink (broadcast) channel, opportunistic power control in the form of channel dependent signal-to-interference-plus-noise ratio (SINR) target setting has been shown to maximize the sum throughput under fairness and sum power constraints [5].

Along another line, several papers suggested that carefully selecting the serving cell (sometimes referred to as link selection) can optimize different objective functions, such as overall system throughput [6], individual quality of service (QoS) targets [7] or other suitable utility functions [8]. Link selection typically operates on a slower time scale (using path loss) than OPC to avoid too frequent handovers and ping-pong effects.

Previous results clearly indicate that joint resource allocation techniques are advantageous, but to the best of our knowledge the throughput-fairness trade-off of the joint link selection and power allocation task has not been addressed previously. For example, a hybrid of centralized and distributed algorithms for subcarrier (i.e., channel) assignment to maximize the overall throughput is proposed in [9]. This scheme has been extended to include power allocation by [10]. Paper [11] studies three adaptive schemes for subcarrier (channel) allocation by means of cross-layer techniques for the purpose of throughput enhancement. Recently, it has been widely recognized that joint allocation of various radio resources has a clear potential over techniques that deal with a single resource, see for example [12], [13], [14] and [15]. However, cell selection is out of the scope of these papers.

Our key observation is that link selection and OPC are both multicell schemes and should complement each others task when exercised appropriately. Therefore, in this paper we examine joint cell (link) and power assignment for the purpose of gaining insight into the gains when these tasks are dealt with jointly.

To this end, we consider two extreme schemes in terms 
of throughput optimization and fairness. The max-min scheme has the objective of maximizing the minimumthroughput (the least happy) user. In contrast, the maximum throughput scheme neglects fairness. As we shall see, it is possible to define an objective function that balances between these extreme cases. Since the joint task turns out to be NP-hard, we use heuristic algorithms to obtain numerical results.

We study these algorithms in orthogonal frequency division multiple access (OFDMA) context by means of a dynamic system level simulator called Rudimentary Network Simulator (RUNE) [16]. RUNE is a suitable tool, since it supports resource allocation algorithms both at the slow and fast time scales and therefore it lends itself to study the gains of joint algorithms [17].

The rest of the paper is structured as follows. The next section describes our multicell model, while Section III formulates both the max-min and maximum throughput problems. Section IV proposes the solution approaches, including a simple reference algorithm and heuristic algorithms for link assignment as well as for power allocation. Section V presents numerical results and Section VI highlights our conclusions.

\section{System Model}

We consider a multicell wireless network consisting of a set $\mathcal{B}$ of base stations (BS), such that each BS maintains the coverage area of its associated cell. In the coverage area of the multicell network, there is the set of mobile stations (MS), denoted by $\mathcal{M}$. To allow for a convenient handling of both the downlink and uplink, we denote the set of information sources by $\mathcal{S}$ and the set of destinations by $\mathcal{D}$. We say that there is a communication link between a MS and its serving BS. Furthermore, we assume that the radio resources that are used in each cell (for example subcarriers, time slots or codes) are orthogonal channels such that there is no intracell interference. In general, a link may comprise multiple channels. The assumption on negligible intracell interference is valid for virtually all major modern telecommunication standards, including orthogonal frequency division multiple access (OFDMA) or orthogonal code division multiple access (OCDMA) schemes and is often used in the literature of multicell models, see for example [18]. Finally, we allow for a complete reuse of all channels in each cell, that is we consider a Reuse-1 system. The sets used in the mathematical formulation are summarized in Table I.

Table I: Definition of the sets of the system model

\begin{tabular}{|cl|}
\hline $\mathcal{S}$ & Set of sources \\
$\mathcal{D}$ & Set of destinations \\
$\mathcal{C}$ & Set of channels per base station \\
$\mathcal{M}$ & Set of mobile users \\
$\mathcal{B}$ & Set of base stations \\
\hline
\end{tabular}

For ease of presentation, in this paper we assume that the basic radio resource is the transmission bandwidth $W$ that is a known given constant. This bandwidth is allocated (and reused) in terms of frequency channels for the communication links. The most important variables and constants of this (rather general) system are summarized in Table II.

Table II: Definition of some variables and constants

$\begin{aligned} x_{i j k} & \begin{cases}1, & \text { if } i \in \mathcal{S} \text { communicates with } j \in \mathcal{D} \\ & \text { on channel } k \in \mathcal{C} \\ 0, & \text { otherwise } \\ 1, & \text { if } x_{i j k}=1 \text { on any channel } k \in \mathcal{C} \\ 0, & \text { otherwise }\end{cases} \\ y_{i j} & \text { the power that source } i \text { uses on channel } k \\ \eta_{i j k} & \text { the throughput between } i \text { and } j \text { on channel } k \\ g_{i j k} & \text { path gain on channel } k \text { of link } i \text { to } j \\ g_{i j} & \text { path gain of link } i \text { to } j, \text { when }|\mathcal{C}|=1 \\ \sigma_{j}^{2} & \text { thermal noise at receiver } j \\ P_{i}^{\text {max }} & \text { the maximum transmit power of sender } i \\ W & \text { transmission frequency bandwidth }\end{aligned}$

The signal-to-interference-and-noise-ratio (SINR) on channel $k$ between source $i$ and destination $j$ is given by

$$
\gamma_{i j k}=\frac{g_{i j k} p_{i k}}{\sigma_{j}^{2}+\sum_{m \in\{\mathcal{S} \backslash\{i\}\}} g_{m j k} p_{m k}}, i \in \mathcal{S}, j \in \mathcal{D}, k \in \mathcal{C} \text {; }
$$

while the throughput on channel $k$ between $i$ and $j$ is given by

$$
\eta_{i j k}=W \log _{2}\left(1+\gamma_{i j k}\right), \quad i \in \mathcal{S}, j \in \mathcal{D}, k \in \mathcal{C} .
$$

\section{Problem Formulation}

Given the model setup, we now introduce a joint cell, channel and power allocation optimization problem.

\section{A. The Max-Min Problem}

We first focus on the max-min problem for the downlink and later discuss how this problem can be generalized to uplink and to include the maximum throughput problem (in both directions). The max-min problem for downlink is stated as follows:

$$
\begin{aligned}
& \underset{\eta, p_{i k}, x_{i j k}, y_{i j}}{\operatorname{maximize}} \eta \\
& \text { subject to } y_{i j} \eta \leq \sum_{k \in \mathcal{C}} x_{i j k} \eta_{i j k}, \quad i \in \mathcal{S}, j \in \mathcal{D} \text {, } \\
& \sum_{i \in \mathcal{B}} y_{i j}=1, \quad j \in \mathcal{M}, \\
& \sum_{j \in \mathcal{M}} x_{i j k} \leq 1, \quad i \in \mathcal{B}, k \in \mathcal{C}, \\
& \sum_{k \in \mathcal{C}} p_{i k} \leq P_{i}^{\max }, \quad i \in \mathcal{S}, \\
& x_{i j k} \leq y_{i j}, \quad i \in \mathcal{S}, j \in \mathcal{D}, k \in \mathcal{C}, \\
& 0 \leq y_{i j} \leq 1, \quad i \in \mathcal{S}, j \in \mathcal{D} \text {, } \\
& x_{i j k} \in\{0,1\}, \quad i \in \mathcal{S}, j \in \mathcal{D}, k \in \mathcal{C} \text {, } \\
& p_{i k} \geq 0, \quad i \in \mathcal{S}, k \in \mathcal{C} \text {. }
\end{aligned}
$$

In this formulation, $\eta$ is an auxiliary variable that is defined by constraint (3b). Constraint (3b) ensures that 
for all active links $\eta$ is less than or equal to the sum of the throughput on the active channels on that link. Maximizing $\eta$ in the objective function (3a) ensures that $\eta$ is equal to the minimum sum throughput on the active links, and that this minimum is maximized. Constraint (3c) ensures that each MS is connected to exactly one BS, while constraint (3d) enforces that each BS at most has one MS per channel. Constraint (3e) is the maximum transmit power constraint. Finally constraints (3f) to (3i) define the variables, previously given in Table II.

The corresponding uplink problem is given by interchanging $i$ and $j$ in constraints (3c) and (3d), they become:

$$
\begin{array}{lrl}
\sum_{j \in B} y_{i j}=1, & i \in M, \\
\sum_{i \in M} x_{i j k} & \leq 1, & j \in B, k \in C,
\end{array}
$$

In [19], the complexity of the joint cell, channel and power allocation problem (3) has been studied and shown to be NP-hard and not approximable unless $\mathrm{P}$ is equal to NP. Therefore, we will consider heuristic methods in the following section.

\section{B. A Generalization of Problem (3)}

Here we introduce a generalization of the problem given in (3). To study the price of fairness, we introduce a parameter $\alpha \in[0,1]$ that will serve as a weight in the objective function between maximizing the minimum throughput among all users and maximizing the total user throughput.

$$
\begin{array}{ll}
\underset{\eta, p_{i k}, x_{i j k}, y_{i j}}{\operatorname{maximize}} & (1-\alpha) \eta+\frac{\alpha}{|\mathcal{M}|} \sum_{i \in \mathcal{S}, j \in \mathcal{D}, k \in \mathcal{C}} x_{i j k} \eta_{i j k} \\
\text { subject to } & \text { constraints }(3 b) \text { to }(3 i) .
\end{array}
$$

In the problem formulation given in (5), the objective can be changed from maximizing the minimum user throughput to maximizing the total system throughput by changing the parameter $\alpha$. By setting $\alpha=0$ the objective becomes to maximizing the minimum user throughput, which gives the same model as (3). While the objective becomes to maximize the total system throughput, when $\alpha=1$.

\section{Solution ApproAch}

\section{A. Separated Link, Channel and Power Allocation}

Because of the complexity of problems (3) and (5), we resort to heuristic algorithms that are based on the decomposition of the problems to the separate tasks of link, channel and power allocation. Furthermore, to avoid modeling the details of scheduling algorithms, we assume that the overall number of MSs does not exceed the (number of BSs) $\times($ number of channels per BS $)$.

\section{B. Link Assignment}

We focus on a link assignment algorithm that gives a feasible cell allocation, i.e., the number of MS at any BS must not exceed the number of channels.
1) Reference Algorithm: For link assignment, we first define a reference algorithm that assigns links according to the largest available path gain. We refer to this algorithm as the Direct Greedy Link Assignment (DGA). In DGA,

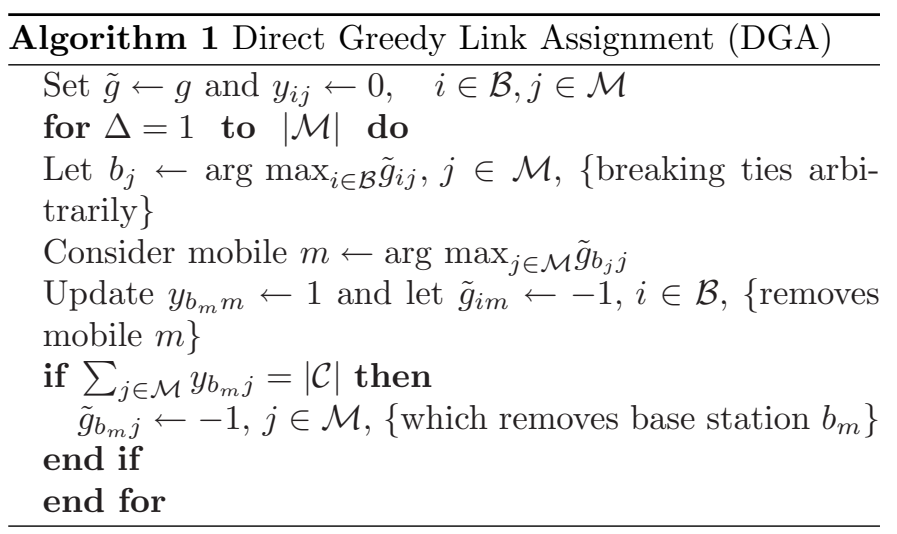

the desired BS for MS $j$ is denoted $b_{j}$. Eventually, the MS with the highest path gain gets assigned to its desired base station. Finally, the variables are updated in order to remove that MS and if the BS has no more channels available the BS is removed from the set of available BSs.

2) Maximize the Total Path Gain Link Assignment (MTGA): In the MTGA approach, the sum of $g_{i j}$ over the transmission links is maximized, which leads to minimizing the path gain over unused links.

$$
\begin{aligned}
\underset{y_{i j}}{\operatorname{maximize}} & \sum_{i \in \mathcal{B}, j \in \mathcal{M}} g_{i j} y_{i j} \\
\text { subject to } & \sum_{i \in \mathcal{B}} y_{i j}=1, \quad j \in \mathcal{M}, \\
& \sum_{j \in \mathcal{M}} y_{i j} \leq|\mathcal{C}|, \quad i \in \mathcal{B}, \\
& y_{i j} \in\{0,1\}, \quad i \in \mathcal{B}, j \in \mathcal{M} .
\end{aligned}
$$

Constraint (6b) ensures that each mobile connects to exactly one base station. Constraint (6c) ensures that BSs do not use more channels than what is available. By relaxing constraint (6d) to $y_{i j} \in[0,1]$, the optimization problem becomes a transportation problem, which is a linear programming problem known to give integer solutions, see, e.g., [20].

3) Maximize the Minimum Path Gain Link Assignment $(M M G)$ : Next, we consider maximizing the minimum path gain (MMG) link assignment that maximizes the smallest $g_{i j}$ over the transmission links.

$$
\begin{aligned}
\underset{\eta, y_{i j}}{\operatorname{maximize}} & \eta \\
\text { subject to } & y_{i j} \eta \leq g_{i j} y_{i j}, \quad i \in \mathcal{B}, j \in \mathcal{M} \\
& \text { constraints }(6 b) \text { to }(6 d) .
\end{aligned}
$$

Maximizing $\eta$ ensures that $\eta$ is equal to the minimum path gain on the assigned links, and that this minimum is maximized. 


\section{Power Allocation}

1) Notation: In line with recent advances in power control [3], [4], we consider a power control scheme that allocates transmission power taking into account small scale fading. We first focus on a max-min formulation and then discuss the max-throughput power control problem. To this end, we introduce some additional notation in Table III.

Table III: Sets and variables used for power control

\begin{tabular}{|l|l|}
\hline $\mathcal{L}=\left\{(i, j): y_{i j}=1, i \in \mathcal{S}, j \in \mathcal{D}\right\}$, & Set of active links \\
$\mathcal{C}_{i j}=\left\{k \in \mathcal{C}: x_{i j k}=1\right\}, \quad(i, j) \in \mathcal{L}$, & Set of active channels \\
$\mathcal{C}_{i}=\bigcup_{j \in \mathcal{D}:(i, j) \in \mathcal{L}} \mathcal{C}_{i j}, \quad i \in \mathcal{S}$, & Set of active channels \\
& by each sender \\
\hline
\end{tabular}

2) The Max-min Power Allocation Problem: The maxmin power allocation problem is formulated as follows

$$
\begin{aligned}
\underset{\eta, \eta_{i j}, p_{i k}}{\operatorname{maximize}} & \eta \\
\text { subject to } & \eta \leq \sum_{k \in \mathcal{C}_{i j}} \eta_{i j k}, \quad(i, j) \in \mathcal{L}, \\
& \sum_{k \in \mathcal{C}_{i}} p_{i k} \leq P_{i}^{\max }, \quad i \in \mathcal{S}, \\
& p_{i k} \geq 0, \quad i \in \mathcal{S}, k \in \mathcal{C}_{i} .
\end{aligned}
$$

This problem is in general neither convex nor approximable unless $\mathrm{P}$ is equal to NP [21]. This means that we may not be able to find a global optimum.

\section{A Simplified Generalization of Problem (8)}

Let us simplify optimization problem (8) by only letting each transmitter use one channel $k \in \mathcal{C}$. We also generalize the objective function to (5a). To simplify notation we denote $p_{i k}$ as $p_{i}, i \in \mathcal{S}$ and $\eta_{i j k}$ as $\eta_{i j}, i \in \mathcal{S}, j \in \mathcal{D}$.

$$
\begin{array}{cl}
\underset{\eta, \eta_{i j}, p_{i k}}{\operatorname{maximize}} & (1-\alpha) \eta+\frac{\alpha}{|\mathcal{M}|} \sum_{(i, j) \in \mathcal{L}} \eta_{i j} \\
\text { subject to } & \eta \leq \eta_{i j}, \quad(i, j) \in \mathcal{L}, \\
& 0 \leq p_{i} \leq P_{i}^{\max }, \quad i \in \mathcal{S} .
\end{array}
$$

With $\alpha=0$, this optimization problem can be treated as convex and is solvable in polynomial time, while with $\alpha=1$, the problem is NP-hard [22]. So, in general one can not expect to solve (9) to global optimality.

\section{Numerical Results}

\section{A. Simulation Environment}

To simulate realistic cellular systems we used the MATLAB based Rudimentary Network Emulator (RUNE). RUNE is available in [16] that also includes a detailed description of the simulator. It can set up a system of base stations, with both omni and directional antennas and distribute users according to some predefined distribution over the coverage area of the system. RUNE can set up realistic path gain matrices including distance dependent path gain, shadow and fast fading, where fast fading is simulated by Rayleigh fading. A typical RUNE simulation session consists of an outer and an inner loop. The outer loop is concerned with generating and terminating users. The inner loop is responsible for mobility and calculating channel coefficients.

We consider a system consisting of $7 \mathrm{BSs}$ and $7 \mathrm{MSs}$ distributed uniformly (unless otherwise stated) in the coverage area. Each base station has a single channel (i.e., assuming that at most one user is scheduled at a time). The main parameters of this system are described in Table IV while Table V details some further parameters relevant for generating the path gain matrix. We solve the power allocation problem using the MATLAB optimization solver fmincon.

Table IV: The main parameters in the system

\begin{tabular}{|r|l|}
\hline Parameter & Value \\
\hline Cell Radius & $500 \mathrm{~m}$ \\
Number of Sectors per Site & 1 \\
Number of Clusters per System & 1 \\
Maximum Power of Mobile & $24 \mathrm{~dB}$ \\
Maximum Power of Base Station & $43 \mathrm{~dB}$ \\
Carrier Frequency & $2 \mathrm{GHz}$ \\
Chunk Bandwidth & $0.2 \mathrm{MHz}$ \\
\hline
\end{tabular}

Table V: Path gain specific parameters

\begin{tabular}{|r|l|}
\hline Parameter & Value \\
\hline Gain at 1 meter Distance & $-28 \mathrm{dBm}$ \\
Noise & $-103 \mathrm{dBm}$ \\
Distance Dependent Path Gain Coefficient & 3.5 \\
Standard Deviation for the log-normal Fading & $6 \mathrm{~dB}$ \\
Log-normal Correlation Downlink & 0.5 \\
Correlation Distance & $110 \mathrm{~m}$ \\
Fast Fading & Rayleigh \\
\hline
\end{tabular}

Our results are mainly based on two different simulations. In the first simulation the system is initialized and run for one outer loop and four inner loops. The users are then given entirely new positions. The procedure is repeated 15 times. We refer to this simulations as the 15 system run (Figures 1 to 4 ). The second simulation has 30 outer loops and four inner loops. Contrary to the 15 system run, the users only move slightly from their previous positions. We refer to this simulations as the 30 second run (Figures 5 and 6 ). Within the 30 second run three different initial user distributions are presented; uniform, close and far (relative to the BSs). 


\section{B. Numerical Results}

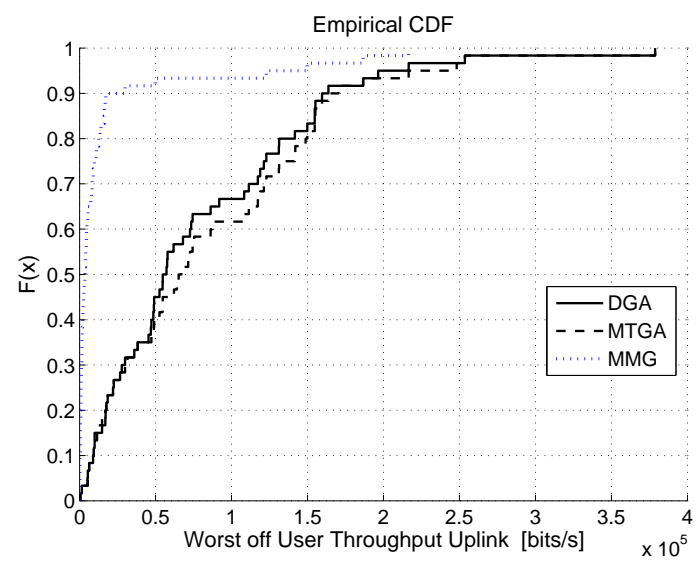

Figure 1: Comparing the performance of the three link assignment algorithms for the max-min power allocation: DGA, MTGA and MMG in terms of the worst off user throughput.

Figure 1 compares the performance of the three link assignments in terms of the throughput received by the user worst off (poorest) in the system.

The worst off user is actually a collection of users, that in terms of least throughput contains the worst off user within each individual time instance. Maximizing the minimum path gain enforces a closely equal throughput distribution, while maximizing the total path gain performs quite similarly to the reference assignment, DGA.

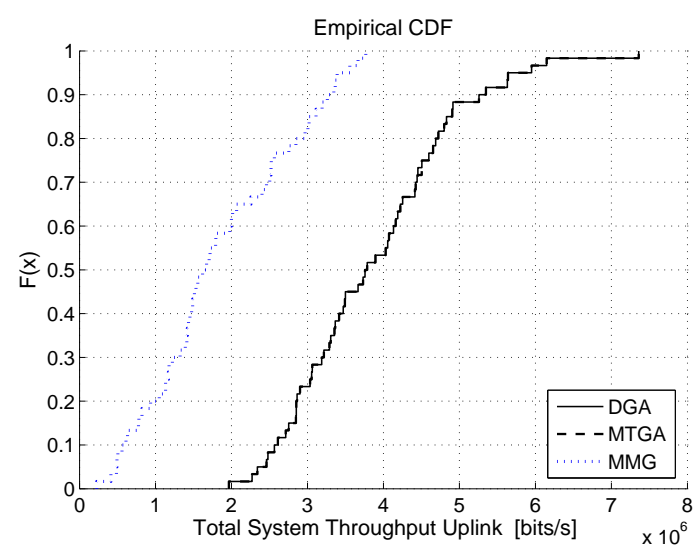

Figure 2: Comparing the performance of the three link assignment algorithms for max-throughput power assignment: DGA, MTGA and MMG in terms of the total throughput.

Figure 2 shows that the fairness of maximizing the minimum path gain by MMG comes at a severe loss in the total system throughput. For example the 50th percentile of the total user throughput is more than twice when using DGA or MTGA. It is interesting to note that DGA and MTGA perform quite similarly, which indicates that DGA is a viable candidate when trying to maximize the total throughput.

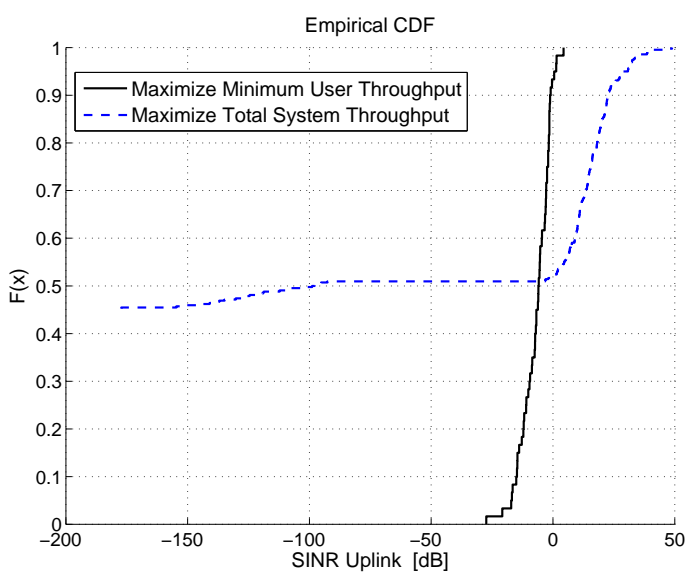

Figure 3: Comparing the performance of the max-min and the maximum throughput power allocation in terms of the uplink SINR distribution at the base stations.

Figure 3 compares the uplink SINR for the max-min and the maximum throughput power allocation schemes (using MTGA link assignment), i.e., solving (8) with $\alpha=0$ and $\alpha=1$ respectively. As expected, the max-min power allocation enforces similar achieved SINR values at the price of keeping all users in a low SINR region (below zero $\mathrm{dB})$. This is in sharp contrast to the maximum throughput power allocation that results in a large SINR spread over users providing very high SINR to some users and none to other users.

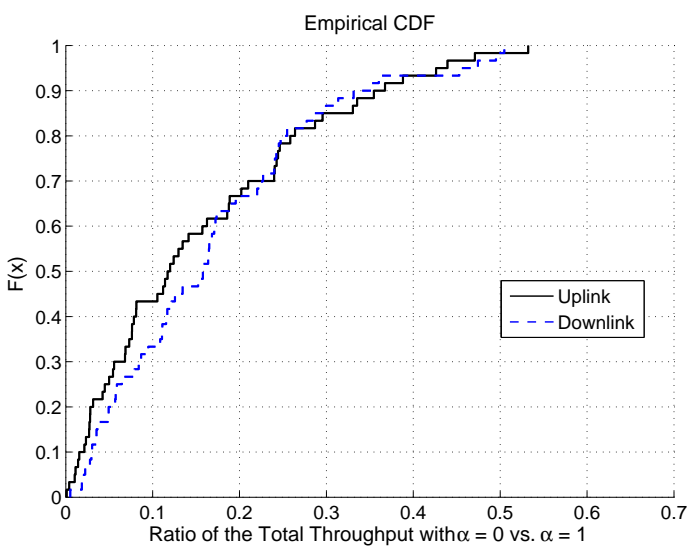

Figure 4: Comparing fair $(\alpha=0)$ and throughput maximizing $(\alpha=1)$ power allocations by plotting the ratio of the associated total throughput values.

Figure 4 shows that the fairness of the max-min power allocation also comes at a high cost in terms of loss in the total system throughput. The max-min allocation $(\alpha=0)$ can hardly ever achieve $50 \%$ of the throughput of the maximum throughput allocation $(\alpha=1)$. The total 
throughput drops at least with $50 \%$ and typically with more than $80 \%$ when allocating power with the max-min power allocation.

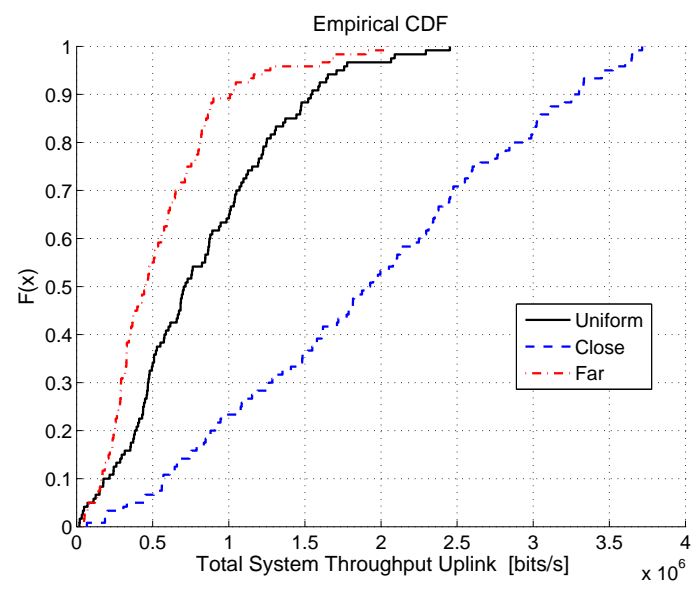

Figure 5: The total system throughput for the max-min allocation in the three user distribution cases "Uniform", "Close", "Far".

Figure 5 shows the impact of the user distributions (close, uniform and far) on the achieved uplink total throughput when employing the max-min power allocation (and MTGA link assignment). Here we note that even with the max-min power allocation, there is a significant spread of the achieved throughput in all three user distribution cases. This result suggests that (even if most users are relatively close to their respective serving base stations), max-min power allocation has only a limited impact on the user throughput fairness.

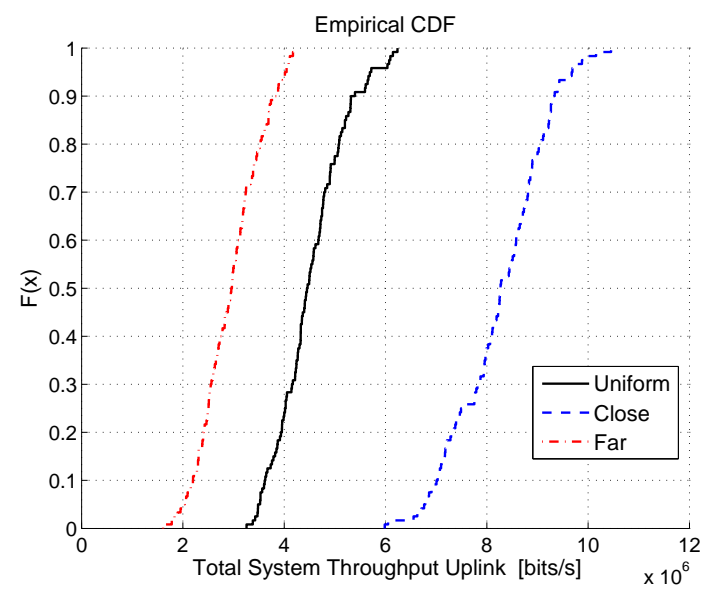

Figure 6: Comparing the total system throughput when employing the maximum throughput power allocation for the three user distribution cases.

Figure 6 compares the total system throughput between the three different distributions, using max-throughput power allocation. Compared with Figure 5, we note a much larger system throughput than with the max-min power allocation (e.g., the 50th percentile of the uniform distribution increasing from $0.75 \mathrm{Mbps}$ to $4.5 \mathrm{Mbps}$ ), without increasing the relative spread of the throughput distributions over the users.

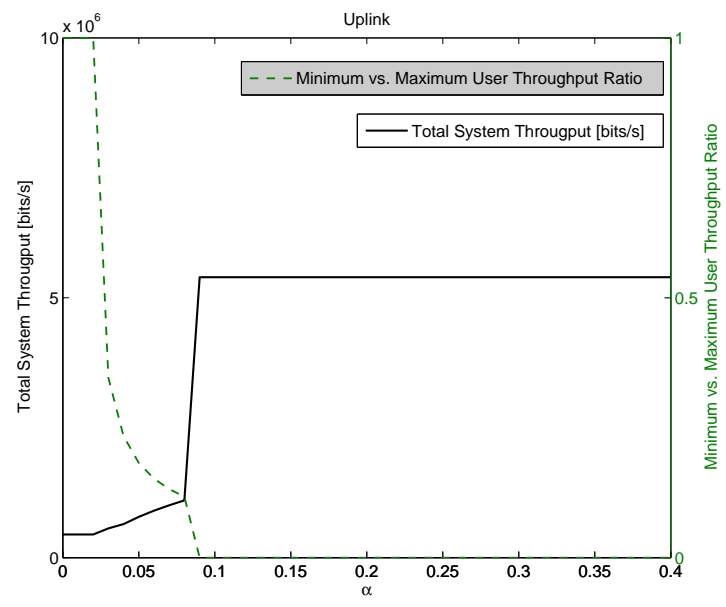

Figure 7: The total system throughput (left axis) for $\alpha$ along with the ratio of the minimum user throughput divided by the maximum user throughput (right axis), in uplink.

In Figure 7 a fixed setup is considered for the uplink, where only the objective function varies by the parameter $\alpha$. In this figure we show how the total system throughput and how the ratio between the minimum user and the maximum user varies as $\alpha$ goes from zero to one (the figure shows the interesting area, $\alpha \in[0,0.4])$. The throughput is equal to that of the optimal solution of the maxthroughput (i.e., $\alpha=1$ ) for a large range of $\alpha$ values. A combination of fairness and maximizing total throughput only takes place on a smaller part of the interval.

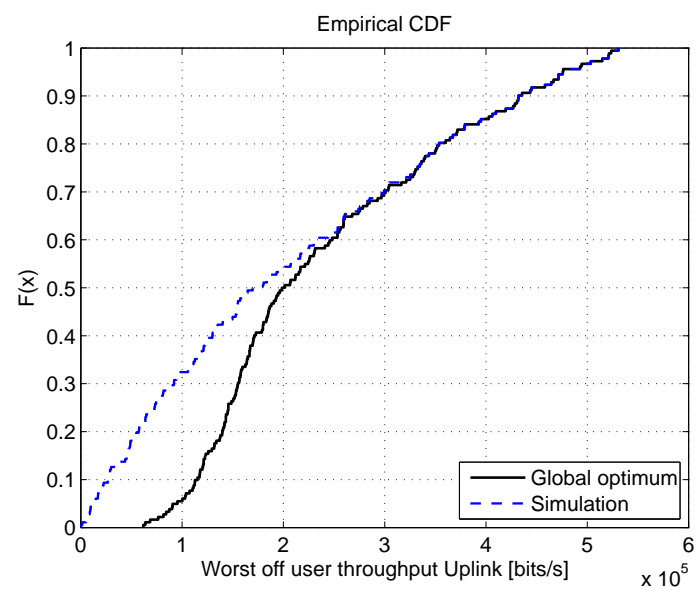

Figure 8: Comparing the worst off user throughput in uplink when employing the max-min allocation simulation to the global optimal solutions. 
Figure 8 shows the worst off user's throughput in terms of global optimum performance and actual performance by our algorithms. One can note that for larger $\eta$ values the algorithms seem to perform close to the global optimum, while for smaller $\eta$ values there is a deviation.

\section{Conclusions}

In this paper we considered the problem of joint serving cell selection (link selection) and power allocation with and without fairness constraints. We formulated this problem as an optimization task, whose complexity motivated to seek heuristic algorithms. We examined the performance of max-min fair and throughput optimizing link and power allocation algorithms by means of system simulations.

We found that a simple link allocation algorithm (termed direct greedy, DGA) that simply relies on the largest available path gain performs very close to a link allocation (maximize total path gain, MTGA) that maximizes the sum path gain. We also compared the impact of power allocation on the total throughput and fairness and found that a max-min power allocation renders virtually all users to the poor SINR domain. Over time we still observed a large spread in the throughput distribution, even when users are close to their respective serving base stations. All users had equal throughput for each fix time instance, but as their location varies so does the throughput of the system. In contrast, a throughput maximizing power allocation dramatically increases the throughput to most users while allocating no power to a few users.

We examined link and power allocations that are "in between" these extreme approaches and found that in a narrow region of the control parameter $\alpha$ it is indeed possible to tune the trade off between throughput and fairness.

Finally, we examine the performance of the algorithms as they maximize the user worst off, by comparing to the global optimum solution. Especially in the lower regions of the worst off user throughput it is possible to improve the performance.

\section{ACKNOWLEDGEMENTS}

The authors are grateful to Anders Forsgren, Johan Håstad and Mikael Prytz for many valuable suggestions.

\section{REFERENCES}

[1] R. Knopp and P. A. Humblet, "Information Capacity and Power Control in Single-cell Multiuser Communications", in the Proc. IEEE Internatinal Conference on Communications, (ICC), Seattle, WA, pp. 18-22, June 1995.

[2] J. Peisa, S. Wager, M. Sågfors, J. Torsner, B. Göransson, T. Fulghum, C. Cozzo and S. Grant: High Speed Packet Access Evolution - Concept and Technologies. Proceedings of the IEEE VTC Spring 2007.

[3] K. Leung and C. W. Sung, "An Opportunistic Power Control for Cellular Network", IEEE/ACM Transactions on Networking, Vol. 14, No. 3, June 2006.

[4] P. Hande, S. Rangan, M. Chiang and X. Wu, "Distributed Uplink Power Control for Optimal SIR Assignment in Cellular" IEEE/ACM Transactions on Networking, pp. 1420-1433, December 2008
[5] N. Reider, G. Fodor and A. Racz, "Opportunistic Target SINR Setting for the MIMO Broadcast Channel", European Wireless '10, Lucca, Italy, May 2010.

[6] H. Zhang and S. Rangarajan, "Joint Load Balancing, Scheduling and Interference Mitigation in Multicell and Multicarrier Wireless Data Systems", Proc. of 7th International Conf. Modeling and Optimization in Mobile, Ad Hoc and Wireless Networks, pp. 49-58, 2009

[7] H. Chaouchi, "QoS-Aware Handover Control in Current and Future Wireless/Mobile Networks", Annals of Telecommunications, Vol. 59, No. 5-6, pp. 731-746, DOI: 10.1007/BF03179695.

[8] A. Sang, X. Wang. M. Madihian and R. D. Gitlin, "A Loadaware Handoff and Cell-site Selection Scheme in Multi-cell Packet Data Systems", Proc. IEEE Globecom, Vol. 6, pp. 39313936, 29 November-3 December 2004.

[9] G. Li and H. Liu, "Downlink Radio Resource Allocation for Multi-cell OFDMA System", IEEE Transactions on Wireless Communications, Vol. 5, No. 12, pp. 3451-3459, December 2006.

[10] Chrysostomos Koutsimanis and Gábor Fodor, "A Dynamic Resource Allocation Scheme for Guaranteed Bit Rate Services in OFDMA Networks", IEEE Interntional Conference on Communications, 2008.

[11] I. Koutsopoulos, L. Tassiulas, "Cross-Layer Adaptive Techniques for Throughput Enhancement in Wireless OFDM-Based Networks", IEEE Transactions on Networking, Vol. 14, No 5, pp. 1056-1066, October 2006

[12] T. Thanabalasingham, S. Hanley, L. L. H. Andrew and J. Papandripoulos, "Joint Allocation of Subcarriers and Transmit Powers in a Multiuser OFDM Cellular Network", IEEE International Conference on Communications '06, pp. 269-274, 2006.

[13] A. Gjendemsjø and D. Gesbert and G. E. Øien and G. Kiani, "Optimal Power Allocation and Scheduling for Two-Cell Capacity Maximization", 2nd Workshop on Resource Allocation in Wireless NETworks, RAWNET, Boston, MA, USA, April 3-7, 2006

[14] S. G. Kiani and D. Gesbert, "Maximizing the Capacity of Large Wireless Networks: Optimal and Distributed Solutions", IST 2006, pp. 2501-2505, July 9-14, 2006.

[15] J-W. Cho, J. Mo, S. Chong, "Joint Network-wide Opportunistic Scheduling and Power Control in Multi-cell Networks", IEEE Symposium on a World of Wireless, Mobile and Multimedia Networks, pp. 1-12, 18-21 June 2007.

[16] J. Zander, S-L. Kim, "Radio Resource Management for Wireless Networks", Artech House Publishers, 2001.

[17] H. Æ. Oddsdóttir, "Near Optimal Joint Channel and Power Allocation Algorithms in Multicell Networks", Master Thesis, Royal Institute of Technology, KTH, Stockholm, June 2010.

[18] Chen, Andrews, Heath, "Uplink Power Control in Spatially Multiplexing Systems", IEEE Transactions on Wireless Communications, 2007.

[19] M. Fallgren, "On the Complexity of Maximizing the Minimum Shannon Capacity in Wireless Networks by Joint Channel Assignment and Power Allocation", IEEE International Workshop on Quality of Service, Beijing, China, 2010.

[20] S. G. Nash, A. Sofer, "Linear and Nonlinear Programming", McGraw-Hill, 1996.

[21] M. Fallgren, "On the Complexity of Maximizing the Minimum Shannon Capacity in Wireless Networks by Joint Channel Assignment and Power Allocation", Tehnical Report, Royal Institute of Technology, TRITA MAT 09 OS 08, December 2009.

[22] Z-Q. Luo, S. Zhang, "Dynamic Spectrum Management: Complexity and Duality", IEEE Journal of Selected Topics in Signal Processing, vol. 2, no. 1, 2008. 